\title{
Convivencia escolar mediante la formación en valores apoyada en las tecnologías de la información y comunicación ${ }^{1}$
}

\section{School coexistence through values training supported by information and communication technologies}

\author{
DOI: http://dx.doi.org/10.17981/cultedusoc.9.1.2018.17
}

Fecha de recepción: 15/05/2018. Fecha de aceptación: 16/08/2018

Hernán Cala-Oliveros ${ }^{2}$ iD

Luz Noriega-Carbonó; Nereida De la Cruz Maestre-Mato; Esther Zapata-Rebolledo; Asunción María Gámez-Pérez; Carmen Cecilia Vives; Enrique Fernández-Camargo; Nilcy Patricia Gonzales-Bernal; María Nigrinis-Irreño; Rodin Rafael Marín-Calderón; Zaira Esther Pedrozo-Conedo; Himelda Beatriz Chávez-Lavalle; Liliana Felipe Villalobos ${ }^{3}$

Para citar este artículo

Cala-Oliveros, H., Noriega-Carbonó, L., De la Cruz Maestre-Mato, N., Zapata-Rebolledo, E., Gámez-Pérez, A., Vives, C., Fernández-Camargo, E., Gonzales-Bernal, N., Nigrinis-Irreño, M. Marín-Calderón, R., Pedrozo-Conedo, Z., Chávez-Lavalle, H. y Felipe, L. (2018). Convivencia escolar mediante la formación en valores apoyada en las tecnologías de la información y comunicación. Cultura. Educación y Sociedad 9(1), 219-227. DOI: http://dx.doi.org/10.17981/cultedusoc.9.1.2018.17

\section{Resumen}

En Colombia existe una preocupación en las instituciones educativas por los altos índices de violencia escolar, por lo cual se hace necesario impartir una formación en valores que permitan al estudiante tomar decisiones asertivas al enfrentarse a una situación conflictiva, fortaleciendo así la convivencia escolar. El estudio busco promover la convivencia escolar mediante la formación en valores apoyada en las tecnologías de la información y comunicación. Está guiado bajo los lineamientos metodológicos de la IEP con un diseño basado en las trayectorias de indagación, la unidad de análisis se conformó por sesenta (60) estudiantes de la institución educativa Thelma Rosa de Arévalo ubicada en el municipio de Zona bananera Magdalena. Los resultados dejaron en evidencia la importancia de implementar nuevas estrategias pedagógicas al aula de clase, observando un impacto positivo en la convivencia escolar de los estudiantes mediante la formación en valores apoyada en el uso de las TIC.

Palabras clave: convivencia escolar, formación en valores, tecnologías de la información y comunicación.

\begin{abstract}
In Colombia there is a concern in educational institutions for the high rates of school violence, which is why it is necessary to provide training in values that allow the student to make assertive decisions when facing a conflictive situation, thus strengthening school coexistence. The study sought to promote school coexistence through training in values supported by information and communication technologies. It is guided under the methodological guidelines of the IEP with a design based on the trajectories of inquiry, the analysis unit was formed by sixty (60) students from the educational institution Thelma Rosa de Arévalo located in the municipality of Magdalena banana zone. The results showed the importance of implementing new pedagogical strategies to the classroom, observing a positive impact on students' school coexistence through the formation of values supported by the use of TIC.
\end{abstract}

Keywords: school coexistence, training on values, information and communication technologies.

\footnotetext{
${ }^{1}$ Este artículo ha sido derivado del Programa de Fortalecimiento de la Cultura Ciudadana y Democrática CT+I a través de la IEP apoyada en TIC en el Departamento de Magdalena: CICLON

${ }^{2}$ Docente de la IED Thelma Rosa de Arévalo y líder del grupo de investigación Enseñar para la vida. Correo de correspondencia: hcalao@ gmail.com

${ }^{3}$ Docentes de la Institución Educativa Distrital Thelma Rosa de Arévalo, Sede principal y miembros del grupo de investigación Enseñar para la vida.
} 


\section{Introducción}

Hoy en día la educación como creadora de futuros generaciones del país. Desde la visión que el conocimiento de los valores y la convivencia escolar concibe riquezas en la formación integral de los alumnos y alumnas. En lo manifestó, nace una discordancia porque se han desgastado debido a causas externas, que rodea al medio escolar como las drogas, alcoholismo, tabaquismo, abandono de los padres. Y si esto se une con la indagación obtenida en los medios de comunicación, se obtiene un resultado de una sociedad disgregada entre sus pares que impacta en la convivencia escolar.

En los últimos años, el mundo actual ha estado viviendo profundas transformaciones en todos los niveles de las estructuras sociales, políticas, económicas, culturales y educativas que lo conforman. Es por ello, que se requiere una educación del humano orientada a prepararlo para la incertidumbre, la comprensión ecológica y la comprensión de la condición humana, lo cual exige lograr la formación de individuos íntegros, con valores y principios firmes esenciales para la convivencia humana.

Lamentablemente, las instituciones educativas reportan un alto índice de violencia escolar, lo cual atenta contra la integridad física y psicológica de los estudiantes, debilitando la convivencia en los planteles educativos, diversos estudios demuestran que es necesario implementar nuevas estrategias pedagógicas que se salgan de los parámetros tradicionales para dinamizar las practicas docentes, por lo cual el estudio tuvo como objetivo promover la convivencia escolar mediante la formación en valores apoyada en las tecnologías de la información y comunicación.
El bullying, matoneo o violencia escolar, son conductas presentadas en el aula, que atentan contra la convivencia, se ha identificado que existen diversos factores que inciden en la prevalencia de conductas violentas, uno de ellos es naturalizar las conductas disruptivas o minimizarlas por parte de los docentes, a veces se cree que si no se presta atención el problema desaparecerá, pero sucede todo lo contrario puede aumentar de intensidad, de esta manera los actos realizados por los alumnos pueden estar siendo reforzados por la tolerancia, pasividad, dificultad en la toma de decisiones y el no establecimiento de reglas claras dentro del salón de clases, (Carrasco, \& Veloz, 2014).

Existen algunos factores que posibilitan el aumento del bullying como la sociedad, donde la exclusión, la discriminación, la inseguridad, así como los niveles de pobreza, pueden convertirse en aspectos desencadenantes de comportamientos observados en otras esferas sociales que se trasladan a la relación con los compañeros en la escuela. En ese sentido, el paso de la niñez a la adolescencia se convierte en una etapa del desarrollo evolutivo esencial, donde el ser humano busca pertenecer a algún lugar, es un periodo de exploración, autoconomiento, y construcción de la identidad. Por lo cual, si la confianza en sí mismo y autoestima se ven debilitadas en el sujeto, también se debilita la capacidad para tomar decisiones y el desarrollo adecuado de las relaciones interpersonales, (Cabezas, \& Monge, 2013).

Es de suma importancia, que la educación que se imparten en las escuelas debe formar y permitir al alumno desenvolverse como persona dentro de la sociedad, tomando en cuenta los valores que se imparten desde el hogar, dado que hoy en día resulta evidente que la diversidad 
y las transformaciones que sufre la educación como revolución tecnológica, parte de las necesidades que la sociedad tiene de la escuela, (Pinto, 2016).

Es así como surge, en la época moderna, la preocupación por una protección integral a los estudiantes, observándolos como sujetos potenciales en nuevas acciones de política legislativa en casi todos los países del mundo, lo que ha generado la consagración de los derechos, a través de preceptos, valores y principios constitucionales, generando un análisis científico. (Herrera, 2013).

La IED Thelma Rosa de Arévalo es una institución conformada por dos sedes debido al número de habitantes de la población y la distribución geográfica del mismo, se encuentra ubicada en la localidad de Varela en el municipio de Zona Bananera, la institución trabaja al ritmo de las exigencias educativas, poniendo en marcha diversos proyectos encaminados a la realización personal de sus estudiantes, tomando a los mismos como el centro del currículo, dirigiendo así actividades encaminadas a la formación integral.

Además, mediante la investigación como estrategia pedagógica (IEP) apoyada en TIC, se promueve la construcción de saber y conocimiento. Puesto que, a través de la ruta metodológica planteadas por esta corriente, se inicia con preguntas que respondan a un problema del contexto y se integren a los contenidos del plan de aula, desarrolladas en un ambiente pedagógico social en el que se propende la promoción de capacidades de investigación en cada uno de los actores. Donde el proceso de formación, implica el acompañamiento docente en el recorrido de las trayectorias de indagación donde se incluyen la cultura ciudadana y democrática integradas en la lectura de contextos fuente de las preguntas, en la recuperación de los valores comunitarios presentes y en las soluciones planteadas a los problemas del entorno de la comunidad educativa.

De esta manera los problemas sociales, como el maltrato físico, verbal, falta de tolerancia, respeto por las diferencias, y la carencia de afecto en que viven los estudiantes, se observa la necesidad de implementar estrategias para darle solución a esta problemática. Todo proceso educativo, además de cumplir unos objetivos previamente establecidos a nivel institucional, pretende una ruta enmarcada en principios y valores familiares ya que es precisamente la institución el segundo hogar de nuestros estudiantes, aprendiendo a aceptar nuestra diferencia, tener claro quiénes somos y mantener el enfoque de a dónde queremos llegar.

La implementación de las TIC ofrece múltiples recursos de apoyo a la enseñanza como materiales didácticos, entornos virtuales, internet, blogs, que permiten dinamizar los contenidos curriculares, enseñando valores y fortaleciendo la convivencia escolar, lo cual contribuirá al desarrollo de habilidades como; creatividad, innovación, entornos de trabajo colaborativo basados en el trabajo en equipo y competencias comunicativas, adaptándose al modelo ecológico como instrumento de examen de las raíces de la violencia (ver figura 1).

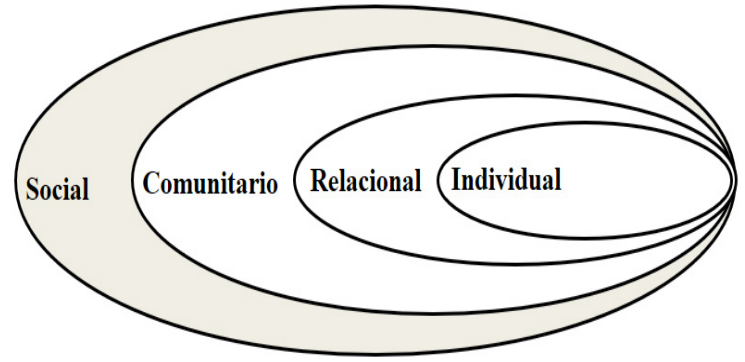

Figura 1. Modelo ecológico como instrumento de examen de las raíces de la violencia Fuente: (Tuvilla, 2015). 
Este modelo ecológico, introducido a fines de los años setenta, se aplicó inicialmente al maltratado de menores, y posteriormente a la violencia juvenil. (Tuvilla, 2015). Los investigadores lo han usado para comprender la violencia doméstica y otro tipo de maltrato. El modelo explora la relación entre los factores individuales y contextuales, y considera la violencia como el producto de muchos niveles de influencias sobre el comportamiento.

Este proyecto nace mediante una observación en el aula, el desvanecimiento de en valores, que cada día se evidencia en el comportamiento de los alumnos y alumnas. Es necesario emprender acciones concretas como: talleres, reflexiones y otras, etc. Al recate de los valores y convivencia escolar para formar alumnos y alumnas capaces de luchar por sus ideales respetado la dignidad del otro. Por ello se hace necesario replantea la formación integral del individuo, profundizando los valores y la convivencia escolar. Tanto en la autoestima, tolerancia, responsabilidad, paz, diversidad, justicia que son los pilares en donde se funden nuestros ideales.

\section{Convivencia escolar mediante la} formación en valores apoyada en las TIC

Hablar de educación en valores y convivencia escolar en este tiempo es interesante, dado la contingencia nacional acerca de la convivencia en comunidad. Además, resultar un tema desafiante del quehacer educativo y social. La convivencia escolar, es un proceso educativo que se desarrolla en la vida diaria del niño y la niña, es por ello, que la misma debe ser considerada de vital importancia para lograr el desarrollo de los contenidos planificados dentro de un aula de clases, de acuerdo a las necesidades e intereses de estos, lo que les favorecerá su propio aprendizaje significativo, (Manzanilla, y Oliveros, 2015).

Dicho de otro modo, los valores expresan las situaciones individuales y colectivas para satisfacer las necesidades humanas porque participan personas en este proceso del quehacer no sólo educativo sino de la sociedad, ahora bien, en el colegio, es donde se trabaja en el tema de los valores a través del manual de convivencia escolar, que es una metodología para el trabajo en valores en el marco de los objetivos transversales de la reforma educativa.

El termino convivencia hace alusión a la relación que se da entre individuos que conviven o que ocupan un mismo espacio de socialización, este término se utiliza particularmente para hablar del hecho de vivir en armonía, tolerando y respetando las diferencias en un ambiente pacífico, donde se respetan las normas y valores que regulan el trato mutuo, (Pick, 2002). Sin embargo, la convivencia escolar es un término que ha sido empleado en el ámbito educativo para disminuir los índices de violencia en la escuela, donde se han implementado prácticas autoritarias que reprimen y castigan a los estudiantes, generando violencia estructural, donde el docente o directivos mantienen una relación vertical, adjudicando al estudiante un rol pasivo como en la educación tradicional, (Carbajal, 2013).

La violencia es una problemática que actualmente se está presentando en todo el mundo, misma que viene a manifestarse en las instituciones educativas, donde recibe el nombre de matoneo escolar, violencia escolar, hostigamiento, acoso escolar, conducta agresiva escolar o bullying, (Garro, 2014). 
El acoso escolar se presenta frecuentemente entre adolescentes, lo que hace que sea trascendental trabajar con este tipo de población, debido a que ellos se encuentran en un período en el que la gente joven experimenta una profunda transición en su desarrollo social, físico e intelectual. Es un tiempo de dudas y auto reflexión, (Hargreaves, Earl y Ryan, 2008). Por tanto, ante entornos conflictivos de esta índole, es muy valioso el aporte que puede dar el personal docente, técnico docente y administrativo, mismo que debería organizarse y plantear estrategias para cambiar las circunstancias, haciendo de la institución educativa un espacio más agradable, (García, 2011).

Por esta razón, es necesario centrar la atención en los colegios, a los cuales asiste gran parte de los adolescentes, lugares en donde se requiere que los estudiantes adquieran una formación apropiada por parte de los educadores y así prevenir que muchos de los educandos sean influenciados por actitudes agresivas que se puedan presentar en sus compañeros, pues en esta etapa de la vida sus sistemas de valores pasan de estar principalmente definidos por sus padres, a verse mucho más influidos por sus compañeros, (Hargreaves, Earl y Ryan, 2008).

Los sistemas educativos inevitablemente están fundados en valores, todas las elecciones que deben hacerse en educación desde los contenidos, la metodología y la formación del docente están basadas en la visión que la sociedad tiene, es decir, en un conjunto de valores. Lo que parece no ocurrir con toda la eficacia es que la sociedad quisiera la apropiación de esos valores por parte de los educandos, en un número creciente de casos están apropiándose de valores o al menos de criterios de juicio y comportamiento, procedentes de otras fuentes extraescolares, entre las cuales los medios de comunicación, cada vez más globalizados, juegan un papel importante, (Pinto, 2016).

El mismo autor, expresa, que el aula coexisten diversos esquemas valorativos presentándose contradicciones e inconsistencias que se manifiestan en la cultura escolar a través del discurso, los comportamientos, actitudes y acciones que se practican pero se le da más importancia a los programas académicos por el saber de los alumnos en términos de competencias intelectuales, descuidando el saber actuar y convivir que se expresa en normas de comportamiento, valores y actitudes ante sí mismo y ante los demás.

Touriñan, (2008), plantea que la Educación en Valores prepara para la convivencia pacífica, porque esta nos lleva al reconocimiento y aceptación del otro en un mundo totalmente globalizado, donde se encuentran diferentes culturas en la vida cotidiana. Y dado que los valores son cognoscibles y estimables, son enseñables, son elegibles y realizables. Por su parte, Garza, \& Patiño, (2000), definen la educación en valores lo hace como un replanteamiento cuya finalidad esencial es humanizar la educación. Los mismos enfatizan que una educación en valores es necesaria para ayudarnos a ser mejores personas en lo individual y mejores integrantes en los espacios sociales en los que nos desarrollamos.

Existen distintos enfoques metodológicos para organizar la práctica educativa a partir de proyectos de trabajo que tienen en la investigación escolar y el empleo de las TIC sus descriptores más relevantes. Las exigencias de la postmodernidad y de culturas globales han traído consigo el diseño de políticas públicas de investigación e innovación de la mano con herramientas tecnológicas que permiten a la sociedad universal el 
acceso a la información y a la construcción de nuevo conocimiento, (Sánchez, \& Ríos, 2013).

La implementación de las tecnologías de la información y la comunicación, en los procesos de enseñanza-aprendizaje y científicos llegan cada vez más a lugares remotos. El uso de diferentes herramientas tecnológicas y de comunicación que han proliferado en los últimos años a través de internet y la incorporación cada vez mayor de diferentes recursos y herramienta de acceso distinto al computador, hacen posible su incorporación en la educación y le han dado una nueva dimensión al aprendizaje.

De hecho, esto tiene que ver con el deseo del ser humano de mantenerse instruido y actualizado a lo largo de su vida no solo a través de escenarios de formación presenciales sino también a través de escenarios de formación por medios virtuales, que les permita acceder a la información y al conocimiento desde cualquier lugar y a cualquier hora con unas condiciones técnicas de acceso básicas, (Romero, \& Quesada, 2014).

En la actualidad las nuevas tecnologías de la información y comunicación (TIC) han tomado un gran auge y su integración al contexto educativo ha generado grandes cambios en el proceso de enseñanza-aprendizaje, estas giran en torno a tres medios básicos: la informática, la microelectrónica y telecomunicaciones; de manera interactiva e interconectadas, lo que permite conseguir nuevas realidades comunicativas, impactando de manera positiva la escuela, (Belloch, 2012).

Las TIC son tecnologías para almacenar, recuperar, procesar y comunicar la información. Existe una variedad de recursos electrónicos que se encuadran dentro del concepto de TIC como; televisión, teléfonos, videos, ordenadores, en- tre otros. Sin embargo, los medios más representativos de la sociedad actual son los ordenadores que nos permiten utilizar diferentes aplicaciones informáticas (presentaciones, aplicaciones multimedia, programas ofimáticos y redes de comunicación, (Belloch, 2012).

Según Ramírez y Ampudia, (2018). La tecnología, es una herramienta que promueve y aumenta las oportunidades de los países en vías de desarrollo a través del conocimiento. En las instituciones educativas estas han tomado fuerza, puesto que la combinación de información, contexto y experiencia, permiten alcanzar los objetivos de las diferentes áreas de estudio, como medio para obtener y administrar el conocimiento requerido en los estudiantes.

Según Avendaño, Cortes \& Guerrero, (2015) expresan que el uso de las tecnologías de la información y la comunicación (TIC) tienen un papel importante en el desarrollo de competencias educativas y sociales, como factores predictores del desempeño académico en estudiantes de básica primaria.

Por otro lado, Herrera, (2016) menciona que las TIC se han convertido en una herramienta innovadora en el contexto educativo generando una relación ineludible que se puede aplicar a cualquier modelo pedagógico a desarrollar y a tener presente de forma trasversal como instrumento pedagógico.

\section{Metodología}

La metodología del estudio se guio a partir bajo los lineamientos de la IEP, con un diseño basado en las trayectorias de indagación, la integración de la IEP apoyada en TIC al aula, articulado al plan de estudios, transversalizando así los procesos de lectura y comprensión lectora a todas las áreas del conocimiento, lo anterior con 
la finalidad de generar una trasformación social y educativa a través del aprendizaje en contexto, involucrando intereses, motivaciones y realidades de los estudiantes para generar conocimiento científico.

\section{Participantes}

La unidad de análisis estuvo conformada por sesenta (60) estudiantes con edades que oscilaron entre los once (11) y catorce (14) años, de los grados sexto y séptimo de bachillerato de la institución educativa Thelma Rosa de Arévalo ubicada en el municipio de Zona bananera Magdalena. cabe destacar que los participantes cumplieron con los requisitos de diligenciamiento del consentimiento informado.

\section{Técnicas e instrumentos}

Utilizando técnicas propias de la investigación cualitativa como; diario de campo y observación participante, técnicas que permitieron registrar la realidad observada, mediante el proceso de investigación realizado, además, estas permitieron sistematizar las experiencias y reflexionar sobre la práctica pedagógica.

\section{Procedimiento}

Se establecieron cinco (5) etapas que especifican la ejecución y las metas trazadas para darle solución a la pregunta problema planteada. Las etapas utilizadas se presentan a continuación.

- Etapa (1): Convocatoria y acompañamiento para la conformación de los grupos de investigación; en esta etapa se seleccionó la muestra para realizar el estudio y posteriormente se realizó la formulación de la pregunta y planteamiento del problema de investigación.
- Etapa (2): Identificación y definición de la temática de investigación, en esta etapa se realizó la fundamentación teórica.

- Etapa (3): Diseño de las trayectorias de indagación, donde se ejecutó la organización del proceso de sistematización, se seleccionaron los instrumentos de recolección de información, se estructuro el diseño metodológico y el proceso de recolección y registro de información.

- Etapa (4): Producción de saber y conocimiento, esta consistió en el análisis de información y redacción del informe de sistematización.

- Etapa (5): Apropiación social del conocimiento, se realizó la divulgación de resultados de investigación en Espacios de apropiación municipal "El maestro tiene la palabra".

\section{Resultados}

Los docentes a partir del proceso de reflexión, sistematizaron las experiencias pedagógicas y diseñaron actividades lúdicas con el apoyo de herramientas TIC, como videos, folletos presentaciones y foros virtuales para fomentar el respeto a diferentes opiniones, para lo anterior se emplearon tres (3) fases que se describen a continuación.

a. Análisis de la problemática de convivencia en la institución.

b. Reflexión sobre las estrategias pedagógicas utilizadas por los docentes.

c. Propuesta pedagógica para dar solución a la problemática a nivel institucional. 
A partir de lo anterior se logró evidenciar que es posible promover la convivencia escolar mediante la formación en valores a través del uso adecuado y responsable de las tecnologías, favoreciendo el desarrollo integral de los estudiantes, haciendo posible su incorporación a la sociedad contemporánea. Articulando las necesidades de formación del individuo con las necesidades sociales. Promoviendo el desarrollo de la creatividad, iniciativa y capacidad para la toma de decisiones.

Por otro lado, es muy importante que las normas de convivencia sean conocidas por todos los miembros de la comunidad educativa y que las medidas adoptadas para sancionar un acto violento cumplan con todas las garantías de derecho y se ejecuten de manera consistente, firme y justa.

\section{Conclusiones}

La sociedad en la que vivimos es caracterizada principalmente por la carencia de valores, reflejando en ella un alto índice de violencia, intolerancia, falta de respeto hacia ellos mismos, hacia las personas, a la naturaleza, etc., sin importar que las autoridades han implementado programas sobre valores e incluso contenidos al respecto en las reformas educativas con la finalidad de coadyuvar en la solución del problema y aun no se obtienen los resultados esperados.

Rescatar los valores para mejorar la convivencia en la comunidad educativa, el proyecto que ha impactado la cultura escolar en la IED Thelma Rosa De Arévalo, a través de los avances significativos obtenidos, a través de una problemática del contexto se concluye que sí es posible realizar investigación como eje transversal a nivel escolar en la cual el nuevo conocimiento es para el estudiante investigador y en cuyo proceso éste adquiere competencias y desarrolla pensamiento científico para enfrentarse a la solución de un problema.

Es muy común encontrara a maestros renegados a salir de su zona de confort y siguen utilizando métodos tradicionalistas, lo más que pueden lograr es formar hombres con poca creatividad, pasivos, que se conforman con lo que hacen o tienen, por eso los valores deben abordarse de acuerdo a la dinámica de la sociedad actual, profundizando en el enfoque formativo de planes y programas actuales como los son el Programa Todos a aprender, computadores para educar, CICLON entre otros. Implementados, atendidos y llevándolos a cabo por los planes de gobierno, para que de esta manera tengan el impacto esperado, y los resultados que se desean obtener. Por ello, los maestros involucrados en la acción educativa deben considerar siempre el contexto en el cual se desenvuelven, atendiendo los sistemas de valores vigentes en la cultura y la sociedad.

Impulsados por las exigencias de la sociedad que nos pide a gritos cambios e innovaciones es preciso tomar conciencia de los problemas de este mundo actual que en gran parte se deben a una crisis de valores, luego buscaremos y coordinaremos en el equipo de maestros líderes y acompañantes, directivos y p comunidad en general para trabajar en forma solidaria y atender más a lo formativo de la educación inculcando o promoviendo los valores.

\section{Referencias bibliográficas}

Avendaño, I., Cortés, O., \& Guerrero, H. (2015). Competencias sociales y tecnologías de la información y la comunicación como factores asociados al desempeño en estudiantes de básica primaria con experiencia de desplazamiento forzado. Diversitas, 11(1), 13-36. 
Belloch. (2012). Las Tecnologías de la Información y Comunicación en el aprendizaje. Universidad de Valencia.

Cabezas, H., \& Monge, M. (2013). Violencia escolar, un problema que aumenta en la escuela primaria costarricense. Revista Electrónica Actualidades Investigativas en Educación, 13 (2).

Carbajal, P. (2013). Convivencia democrática en las escuelas. Apuntes para una reconceptualizacion. Revista Iberoamericana de Evaluación Educativa, 6 (2), 13-35.

Carrasco, M y Veloz, A. (2014). Aprendiendo valores desaprendiendo violencia, un estudio con niñas y niños de escuelas de educación básica en el estado de hidalgo. Ra Ximhai, 10 (7).

García, R. (2011). La violencia escolar. En Educainnova magazine, (13), 40-44.

Garro, J. (2014). Estrategias didácticas y lúdicas para abordar el tema de acoso escolar con los estudiantes de noveno año del Liceo Dr. José María Castro Madriz.

Garza, J y Patiño, S. (2000). ¿Para qué educar en Valores? Documento en línea: http://servicio.bc.uc.edu.ve/multidisciplinarias/educacion-en-valores/ v1n7/v1n72007- 11.pdf

Hargreaves, A; Earl, L y Ryan, J. (2008). Una educación para el cambio: reinventar la educación de los adolescentes. Barcelona, España: Ediciones Octaedro, S.L.

Herrera, B. (2013). La constitucionalización de los derechos del consumidor en Colombia: un análisis desde los derechos sociales fundamentales. Civilizar. Ciencias Sociales y Humanas, 13 (25), 33-47.
Herrera, B. (2016). Cultura Ciudadana y las Tecnologías de la Información y la Comunicación. Editorial YOYOBIZ. Barranquilla-Colombia.

Manzanilla, L, y Oliveros, A. (2015). Educación en valores para el mejoramiento de la convivencia escolar: Universidad de Carabobo. Facultad de Ciencias de la Educación. Febrero 2015 (Bachelor's thesis).

Pick, S. (2002). Formación civica y étical Civics and Ethics. Editorial Limusa.

Pinto, R. (2016). La importancia de promover los valores del hogar hacia las escuelas primarias. Ra Ximhai, $12(3)$.

Ramírez, R., \& Ampudia, D. (2018). Factores de Competitividad Empresarial en el Sector Comercial. Revista Electrónica de Ciencia y Tecnología del Instituto Universitario de Tecnología de Maracaibo (RECITIUTM). Vol. 4, $N^{\circ}$ 1. Pág.: 16-32.

Romero, M., \& Quesada, A. (2014). Nuevas tecnologías y aprendizaje significativo de las ciencias. Enseñanza de las Ciencias, 32 (1), 0101-115.

Sánchez, J., \&Ríos, L. (2013). Uso de las TICS (SCILAB Y WIRIS) y su influencia en el rendimiento en el Álgebra Lineal de los Alumnos del Primer Nivel de Ingeniería de la Escuela Politécnica del Ejército Extensión Latacunga.

Tuvilla, J. (2015). Educación en Derechos Humanos - Hacia una perspectiva global. $2^{\text {da }}$ edición. Pienso (Edición digital).

Touriñan, J. (2008). Educación en valores, educación intercultural y formación para la convivencia pacífica. 